\title{
Prevalence and Risk Factor Analysis of Constipation After Thoracolumbar Vertebral Compression
} \section{Fractures}

\author{
Heng Yin ${ }^{1, *}$ \\ Gaoxiang Wang ${ }^{2, *}$ \\ Jianwei Wang' \\ Yong $\mathrm{Ma}^{2}$ \\ Mao $\mathrm{Wu}^{\mathrm{l}}$ \\ Songming $\mathrm{Qiu}^{2}$ \\ Qiuju $\mathrm{Su}^{3}$
}

'Department of Orthopedics Wuxi Traditional Chinese Medicine Hospital affiliated to Nanjing University of Chinese Medicine, Wuxi, 2 I407I, People's Republic of China; ${ }^{2}$ Nanjing University of Chinese Medicine, Nanjing, 210023. People's Republic of China; ${ }^{3}$ Department of Rehabilitation \& Acupuncture, Wuxi People's Hospital Affiliated Nanjing Medical University, Wuxi, 2I 4023,

People's Republic of China

*These authors contributed equally to this work

\begin{abstract}
Objective: To analyze the prevalence and risk factors of constipation after thoracolumbar vertebral compression fractures (TVCFs).

Methods: This retrospective study reviews the records of patients hospitalized between January 1, 2017 and December 31, 2018 with TVCFs. A total of 117 patient's records are included $(n=117)$. Univariate and multivariate analysis using the logistic regression method are carried out to identify the prevalence and potential risk factors for constipation after TVCF, including gender, age, number of fractured vertebrae, major segment of vertebral fracture, degree of compression, use of painkillers, diabetes, and the intervention of Zengyechengqi decoction.
\end{abstract}

Results: Among the 117 patients with TVCFs that were included in this study, 83 (70.9\%) patients developed constipation. Univariate analysis showed that the factors of degree of vertebral compression and the preintervention of Zengyechengqi decoction had statistically significant effects on the incidence of constipation after TVCF $(\mathrm{P}<0.05)$, indicating that they might contribute to the incidence of constipation after TVCF. Multivariate logistic regression analysis showed that degree of vertebral compression was a risk factor $(\mathrm{P}<$ 0.05), while preintervention of Zengyechengqi decoction was a protective factor $(\mathrm{P}<0.05)$, for constipation after TVCF.

Conclusion: Patients with vertebral fractures featuring a higher degree of compression may have a higher risk of constipation. Preintervention of Zengyechengqi decoction can reduce the incidence of constipation after TVCF.

Keywords: constipation, degree of vertebral compression, risk factors, thoracolumbar vertebral compression fractures, Zengyechengqi decoction

\section{Introduction}

Thoracolumbar vertebral compression fractures (TVCFs) have a high incidence in the elderly population. ${ }^{1}$ For every 100 people of more than 70, 20 new vertebral osteoporotic fractures occurred each year. ${ }^{2}$ Constipation is one of the most common complications after TVCF, which may induce abdominal distention, appetite loss, nausea, other uncomfortable symptoms, and even progressive aggravation of constipation, drug dependence, internal water and electrolyte imbalance, intestinal obstruction, intracranial hemorrhage, thrombus shedding, and myocardial infarction. ${ }^{3}$ When constipation occurred after TVCFs, the most commonly used drugs include lactulose, polyethylene glycol, senna, etc., lactulose and polyethylene glycol have a too slow effect; The dosage of senna is difficult to control, which can
Correspondence: Qiuju Su Department of Rehabilitation \& Acupuncture, Wuxi People's Hospital Affiliated Nanjing Medical University, No. 299 of Qingyang Road, Wuxi, 214023, People's Republic of China

Tel +8615961817218

$\mathrm{Fax}+86510-82700775$

Email suqiuju_336@I63.com 
easily cause severe diarrhea and aggravate the discomfort of patients. None of these drugs are suitable for constipation prevention after TVCFs. The data on the prevalence and risk factors of constipation after TVCF are limited. It is extremely important to provide preventive measures to improve the patient's quality of life during fracture treatment.

Zengyechengqi decoction is one of the most famous traditional Chinese medicine (TCM) decoctions; it was first used in the classic TCM book Analysis of Heart Diseases. It consists of rhubarb, mirabilite, rehmanniae, scrophulariae, and radix ophiopogonis. Zengyechengqi decoctions are mainly used for constipation and abdominal distension and have been used for more than 300 years. ${ }^{4}$ Rhubarb and mirabilite are purgative, while scrophulariae and radix ophiopogonis nourish Yin and blood, playing the positive role of strengthening the body's resistance. ${ }^{5}$ Zengyechengqi decoction can enable Qi, blood and fluid recovery in the body, that is, maintain the physiological barrier of the body and remove pathological products, such as toxic heat, phlegm, dampness, and blood stasis. Zengyechengqi decoction is also used to treat irritable bowel syndrome and senile constipation. ${ }^{6}$

In this study, we retrospectively analyzed the demographic and clinical characteristics of patients with TVCF to identify the prevalence and potential risk factors for constipation after TVCF; these data included gender, age, number of fractured vertebrae, major segment of vertebral fracture, degree of compression, use of painkillers, diabetes, and the intervention of Zengyechengqi decoction. The purpose of the article is to analyze the prevalence and risk factors of constipation after TVCFs, and explore the effect of the traditional Chinese medicine Zengyechengqi decoction.

\section{Materials and Methods \\ Data Collection}

The medical records of 309 hospitalized patients between January 1, 2017 and December 31, 2018 with TVCF were reviewed. The inclusion criteria were: (1) age $\geq 60$ years old, (2) a history of trauma, (3) hospitalization within 24 hours of fracture, and (4) diagnosed with TVCF. The exclusion criteria were: patients (1) with other tissue injuries, such as transverse process fracture, pelvic fractures, etc. have been excluded, (2) with tumors, colon polyps, other intestinal organic lesions, or other gastrointestinal tract diseases, (3) who had been treated by a therapist who affects gastrointestinal motility, (4) taking any other medication (except painkillers) that may cause constipation, (5) with spinal cord injury, and (6) with incomplete medical records. Finally, a total of 117 patients who met these criteria were enrolled and their clinical data, including gender, age, number of fractured vertebrae, the main fracture segment, the degree of vertebral compression, TCM syndrome type, use of painkillers, diabetes, use of TCM Zengyechengqi decoction intervention, and the occurrence of constipation, were analyzed. This study was approved by the Ethics Committee of Wuxi TCM Hospital, and the Clinical trial registration number is ChiCTR2000038332. This study complies with the Declaration of Helsinki. All of the included patients gave their written informed consent.

\section{Diagnostic Criteria of TVCF}

The diagnosis of TVCF was based on Mauch et al: ${ }^{7}$ (1) patients with a history of trauma, (2) patients with symptoms of back or low back pain, (3) patients with obvious tenderness and pain on percussion of back or low back; (4) X-ray and CT images revealing TVCF; and (5) MRI revealing a recent vertebral compression fracture: Most of the recent vertebral compression fractures show wedgeshaped vertebral bodies on MRI (A few fractures have no wedge-shaped), with low signal changes on T1W1 and high signal changes on T2W2 images.

Some patients received conservative treatment. And some patients received percutaneous kyphoplasty. The indication for the operation: (1) Symptomatic vertebral angioma. (2) Painful vertebral body tumors and acetabular tumors. (3) Severe painful osteoporosis with loss of height and/or with compression fractures of vertebral bodies.

\section{Diagnostic Criteria of Constipation}

The diagnosis of constipation was based on the Rome IV criteria: ${ }^{8}$ patients with (1) symptoms of lumpy or hard stools, (2) patients with symptoms of a prolonged period between bowel movements, ie, > 2-day interval; and (3) patients with extremely dry excrement, accompanied by symptoms of fatigue, intolerance, and other discomforts.

\section{Classification of Vertebral Compression Degree}

The criteria were based on the Genant image classification, ${ }^{7}$ compared with the height of the relevant vertebra before fracture: (1) mild compression, 20\% 
$25 \%$ degree of vertebral compression; (2) moderate compression, $25 \% \sim 40 \%$ degree of vertebral compression; and (3) severe compression, $>40 \%$ degree of vertebral compression.

\section{Diagnosis of TCM Syndrome}

According to the medical records, two types of TCM syndrome were included: Qi stagnation/blood stasis syndrome and liver/kidney insufficiency syndrome. According to the TCM syndrome type standard, ${ }^{9}$ Qi stagnation/blood stasis syndrome of TVCF was mainly manifested by pain in the lumbar region, fixed pain position, bruising of the back especially in the lumbar region, a dark red or thinly coated tongue, and an astringent pulse string. Liver/kidney insufficiency syndrome was mainly manifested by mild lumbar pain, pain when turning over, weakness of the waist and knees, dry eyes, a pale white or dry red tongue, and a weak pulse.

\section{Zengyechengqi Decoction Treatment}

The composition of the Zengyechengqi decoction was as follows: scrophularia (30g), radix ophiopogonis (24g), rehmanniae $(24 \mathrm{~g})$, rhubarb $(9 \mathrm{~g})$, and mirabilite $(4.5 \mathrm{~g})$. The decoction was prepared by boiling the crude herbs in $2000 \mathrm{~mL}$ of water until the water reduced to $500 \mathrm{~mL}$ as previously described. ${ }^{10,11}$ Then, the decoction was filtered and divided into two doses $(250 \mathrm{~mL}$ each). According to the traditional Chinese medicine, Zengyechengqi decoction could be used for patients who meet the following criteria: 1) aged 50-85 years old, 2) slow movement, 3) speak in a low voice, 4) limited joint mobility, 5) not eating or drinking enough, 6) red-colored tongue, 7) constipation. All patients who were given Zengyechengqi decoction in this study met the above-mentioned criteria. They were given the decoction twice a day $(250 \mathrm{~mL}$ each time) from the first day of their admission.

\section{Statistical Analysis}

SPSS 25.0 software was used for the data collation, statistics and analyses. A $\chi^{2}$ test was used for univariate analysis of each factor, and $\mathrm{P}<0.05$ was used to screen for the possible correlation factors with statistical significance under a single-factor action. Multivariate logistic regression analysis was used to screen for the possible correlation factors after univariate analysis, and, again, $\mathrm{P}<0.05$ was considered as statistically significant.

\section{Results}

\section{Demographic and Clinical Characteristic} of Patients with Constipation After TVCF

All patients included in this study were tested for neurological deficits and the results showed that their neurological function was normal. The trauma mechanism of all these patients was osteoporotic fracture. The patients $(n=29)$ subjected to surgical treatment received percutaneous kyphoplasty within 3D. The patients who complained severe pain were given non-steroidal anti-inflammatory drugs, such as loxoprofen sodium, diclofenac sodium, or aceclofenac. Data from 117 cases of TVCF were analyzed using the $\chi^{2}$ test. The results showed that the factors of degree of vertebral compression and the preintervention of Zengyechengqi decoction both had a significant impact on constipation after TVCF (P $<0.05$ ), which may be directly related to the onset of the constipation (Table 1).

\section{Multivariate Binary Logistic Regression Analysis of Constipation After TVCF}

According to the results of the univariate analysis, the fracture compression degree and the preintervention of Zengyechengqi decoction may influence the incidence of constipation after TVCF $(\mathrm{P}<0.05)$. These two factors were included in the multivariate binary logistic regression analysis. The results show that the factors of vertebral compression degree and the preintervention factor of liquid and airbearing decoction were independent influencing factors of constipation after TVCF $(\mathrm{P}<0.05)$. Vertebral compression degree is a risk factor $(\mathrm{P}<0.05)$, while the preintervention factor of Zenglizhenqi decoction is a protective factor of constipation after TVCF $(\mathrm{P}<0.05)$. (Table 2, Figure 1)

\section{Discussion}

Constipation is one of the most common complications after TVCF, with an incidence of $36 \%-63 \%{ }^{3}$ It often leads to abdominal distention, appetite loss, nausea, and, in severe cases, even intestinal obstruction, intracranial hemorrhage, and myocardial infarction. ${ }^{7}$ The previous literature has considered the causes to be long-term bed rest, hematoma compression, psychological factors, and diet, among others. ${ }^{12-14}$ According to our study, constipation occurred in 83 cases, with an incidence rate of $70.94 \%$. Therefore, it is important to take advance preventive measures for the population at high-risk of constipation after TVCF. Gender, age, number of fractured vertebrae, major segment of vertebral fracture, degree of compression, use 
Table I Demographic and Clinical Characteristic of Patients with Constipation After TVCF ( $\mathrm{n}=$ II7)

\begin{tabular}{|c|c|c|c|c|c|}
\hline & $\begin{array}{c}\text { Total Number of } \\
\text { Patients (\%) }\end{array}$ & $\begin{array}{l}\text { Number of } \\
\text { Constipation } \\
\text { Patients (\%) }\end{array}$ & $\begin{array}{c}\text { Number of } \\
\text { Patients without } \\
\text { Constipation (\%) }\end{array}$ & $\chi^{2}$ & $P$ value \\
\hline Patients (n) & II (100\%) & 83 (70.9\%) & 34 (29.1\%) & & \\
\hline $\begin{array}{l}\text { Gender } \\
\text { Female } \\
\text { Male }\end{array}$ & $\begin{array}{l}96(82.1 \%) \\
21(17.9 \%)\end{array}$ & $\begin{array}{l}68(81.9 \%) \\
15(18.1 \%)\end{array}$ & $\begin{array}{c}28(82.4 \%) \\
6(17.6)\end{array}$ & $\begin{array}{l}0.003 \\
0.952\end{array}$ & $\begin{array}{l}0.957 \\
0.621\end{array}$ \\
\hline $\begin{array}{l}\text { Age } \\
\qquad \begin{array}{l}60 \sim 69 y \\
70 \sim 79 \text { y } \\
\geq 80 \text { y }\end{array}\end{array}$ & $\begin{array}{l}66(56.4 \%) \\
36(30.8 \%) \\
15(12.8 \%)\end{array}$ & $\begin{array}{c}48(57.8 \%) \\
26(31.3 \%) \\
9(10.8 \%)\end{array}$ & $\begin{array}{l}18(53.0 \%) \\
10(29.4 \%) \\
6(17.6 \%)\end{array}$ & & \\
\hline $\begin{array}{l}\text { Number of fractured vertebrae } \\
\quad \begin{array}{l}\text { I } \\
2 \\
\geq 3\end{array}\end{array}$ & $\begin{array}{c}98(83.8 \%) \\
10(8.5 \%) \\
9(7.7 \%)\end{array}$ & $\begin{aligned} 70 & (84.3 \%) \\
7 & (8.4 \%) \\
6 & (7.2 \%)\end{aligned}$ & $\begin{array}{c}28(82.4 \%) \\
3(8.8 \%) \\
3(8.8 \%)\end{array}$ & 0.095 & 0.953 \\
\hline $\begin{array}{l}\text { Main fracture segment } \\
\text { Thoracic segments (TI0 and above) } \\
\text { Thoracic-lumbar segment (TII L L2) } \\
\text { Lumbar segment (L3 and below) }\end{array}$ & $\begin{array}{c}5(4.3 \%) \\
93(79.5 \%) \\
19(16.2 \%)\end{array}$ & $\begin{array}{c}3(3.6 \%) \\
67(80.7 \%) \\
13(15.7 \%)\end{array}$ & $\begin{array}{c}2(5.9 \%) \\
26(76.5 \%) \\
6(17.6 \%)\end{array}$ & 0.404 & 0.817 \\
\hline $\begin{array}{l}\text { Degree of vertebrae compression } \\
\text { Mild } \\
\text { Moderate } \\
\text { Severe }\end{array}$ & $\begin{array}{l}42(35.9 \%) \\
46(39.3 \%) \\
29(24.8 \%)\end{array}$ & $\begin{array}{l}20(24.1 \%) \\
37(44.6 \%) \\
26(31.3 \%)\end{array}$ & $\begin{array}{c}22(64.7 \%) \\
9(26.5 \%) \\
3(8.8 \%)\end{array}$ & 18.019 & $0.000 *$ \\
\hline $\begin{array}{l}\text { TCM syndrome type } \\
\text { Qi stagnation/blood stasis } \\
\text { Liver/kidney insufficiency }\end{array}$ & $\begin{array}{l}106(90.6 \%) \\
I 1(9.4 \%)\end{array}$ & $\begin{array}{c}78(94.0 \%) \\
5(6.0 \%)\end{array}$ & $\begin{array}{c}28(82.4 \%) \\
6(17.6 \%)\end{array}$ & 2.583 & 0.108 \\
\hline $\begin{array}{l}\text { Use of painkillers } \\
\text { No } \\
\text { Yes }\end{array}$ & $\begin{array}{l}54(46.2 \%) \\
63(53.8 \%)\end{array}$ & $\begin{array}{l}38(45.8 \%) \\
45(54.2 \%)\end{array}$ & $\begin{array}{l}16(47.1 \%) \\
18(52.9 \%)\end{array}$ & 0.016 & 0.900 \\
\hline $\begin{array}{l}\text { Diabetes } \\
\text { No } \\
\text { Yes }\end{array}$ & $\begin{array}{l}102(87.2 \%) \\
15(12.8 \%)\end{array}$ & $\begin{array}{l}73(88.0 \%) \\
10(12.0 \%)\end{array}$ & $\begin{array}{c}29(85.3 \%) \\
5(14.7 \%)\end{array}$ & 0.007 & 0.932 \\
\hline $\begin{array}{l}\text { Use of Zengyechengqi decoction } \\
\text { No } \\
\text { Yes }\end{array}$ & $\begin{array}{l}90(76.9 \%) \\
27(23.1 \%)\end{array}$ & $\begin{array}{l}73(88.0 \%) \\
10(12.0 \%)\end{array}$ & $\begin{array}{l}17(50 \%) \\
17(50 \%)\end{array}$ & 19.570 & $0.000 *$ \\
\hline
\end{tabular}

Note: $* P<0.05$.

of painkillers, and diabetes are potential related factors for constipation. ${ }^{15}$ The use of TCM Zengyechengqi decoction may also be an important factor in constipation. Thus, we included these factors in the univariate analysis. The results suggested that the degree of vertebral compression and preintervention of TCM Zengyechengqi decoction had a significant impact on constipation $(\mathrm{P}<0.05)$, suggesting that they were possible related factors of constipation. The preintervention factor of Zengyechengqi decoction is a protective factor of constipation after TVCF $(\mathrm{P}<0.05)$ that is negatively correlated with the occurrence of constipation.

Subsequently, the degree of vertebral compression was included in the multivariate logistic regression analysis. The results showed that degree of vertebral compression was a risk factor for constipation after TVCF $(\mathrm{P}<0.05)$. The heavier the degree of vertebral compression, the higher the risk of constipation. This may be due to retroperitoneal hematoma after fracture. On vertebral body compression, the vessels in the vertebral body are prone to damage, rupture, and hemorrhage, 
Table 2 Multivariate Analysis of Possible Related Factors

\begin{tabular}{|l|c|c|c|c|}
\hline Factors & $\boldsymbol{\beta}$ & OR & $\mathbf{9 5 \%} \mathbf{C l}$ & $\boldsymbol{P}$ value \\
\hline Mild vertebrae compression & & & & - \\
Moderate vertebrae compression & 1.415 & 4.118 & $1.487 \sim 11.401$ & $0.006^{*}$ \\
Severe vertebrae compression & 1.989 & 7.311 & $1.802 \sim 29.660$ & $0.005^{*}$ \\
Use of Zengyechengqi decoction & -1.789 & 0.167 & $0.061 \sim 0.459$ & $0.00 I^{*}$ \\
\hline
\end{tabular}

Note: $* p<0.05$.

forming a hematoma that compresses the posterior peritoneum, leading to compression of the posterior peritoneum, resultant uplift of the torus ligament, and consequently compression of the duodenum. ${ }^{16}$ The sympathetic ganglia are located anteriorly and bilaterally to the spine. After the occurrence of a vertebral compression fracture, a concomitant hematoma would continue to stimulate the sympathetic ganglia, which would prolong sympathetic nerve stimulation, thereby inhibiting gastrointestinal activity, leading to difficulty in excretion of stool and subsequent constipation.

Moreover, we also found that the preintervention factor of Zengyechengqi decoction was a protective factor for constipation after TVCF $(\mathrm{P}<0.05)$. Zengyechengqi decoction is a well-known TCM decoction that is used for constipation and abdominal distension. According to TCM theory, when compression of the vertebral body occurs, the body undergoes traumatic stimulation, and the vital Qi is therefore relatively deficient. The use of diarrhea medicine to treat constipation can easily cause further damage to the vital Qi, which is a secondary detriment to the body. ${ }^{17}$ Some drugs should be added simultaneously to help maintain the vital Qi, fulfilling the dual roles of intervention and alleviation. Wang et $\mathrm{al}^{18}$ set up a model of heat-clotted intestinal syndrome constipation in rats and found that Zengyechengqi decoction repaired the normal physiological structure of the intestinal tissues, enhanced intestinal immunity, and reduced the amount of inflammatory substances, such as IL-6, IL-1 and TNF- $\alpha$, in the serum. This experiment proved that Zengyechengqi decoction had a definite therapeutic effect on constipation in an animal model. Peng et $\mathrm{al}^{19}$ found that ZengyeChengqi Decoction could enhance the antioxidant capacity of elderly mice with constipation of deficiency of Yin fluid and body fluid and had the effect of anti-oxidative stress. Jiang and Deng $^{20}$ found that Zengyechengqi decoction can improve the imbalance of intestinal flora in rats with intestinal flora disorder, reduce the content of serum inflammatory factors, and regulate the secretion of neuropeptides. Xin and Zhang ${ }^{21}$ showed that the treatment of Zhengyishengqi decoction relieved the clinical symptoms in $90 \%$ of the patients with chronic constipation. Zhang ${ }^{22}$ found that approximately $90.7 \%$ of elderly patients with constipation after femoral neck and intertrochanteric fractures could obtain significant relief from constipation after oral treatment with Zengyechengqi decoction. Chen ${ }^{23}$ treated patients with constipation caused by opioids with Zengyechengqi decoction or lactulose for seven days and found that Zengyechengqi

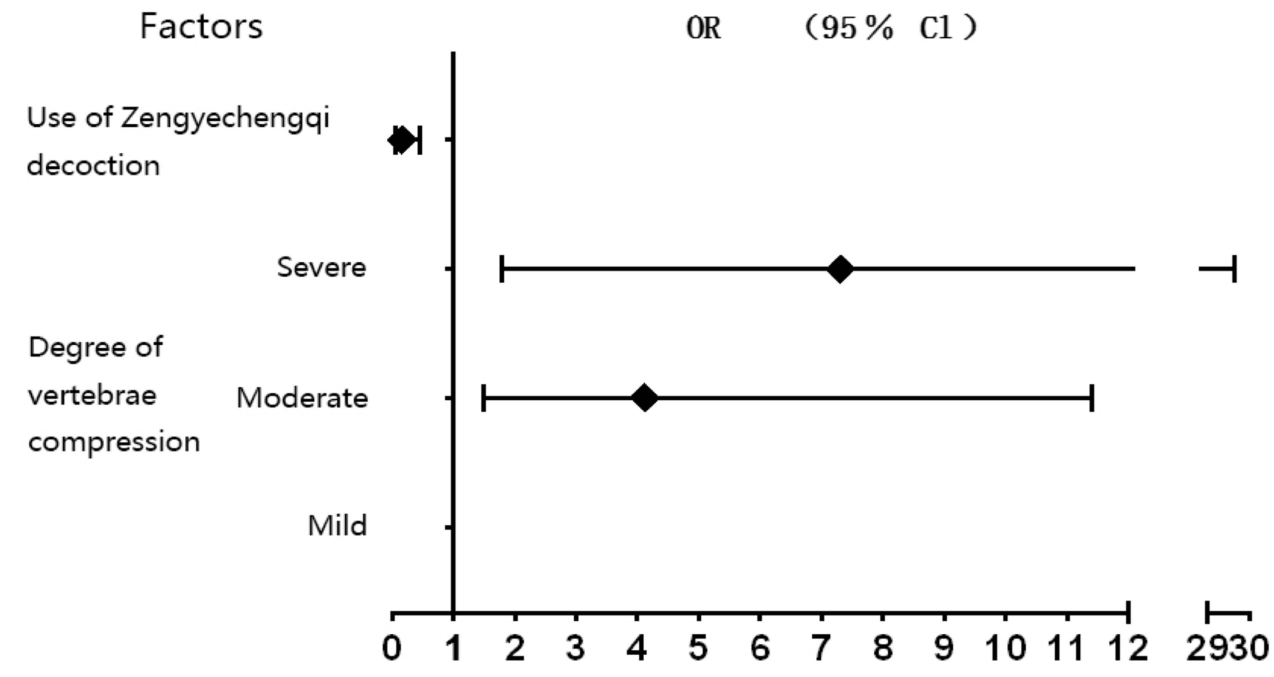

Figure I Multivariate analysis of possible related factors. 
decoction was effective in $94.4 \%$ of the patients, and lactulose was effective in only $81.5 \%$; there was a statistical difference in the efficacy between these two groups. The above findings indicate that Zengyechengqi decoction has a definite therapeutic effect on constipation in patients who are suffering with this condition. Consistently, our data showed that the preintervention of Zengyechengqi decoction had a beneficial effect on prevention of constipation in patients with TVCF.

There are few reports on the risk factors of constipation after thoracolumbar fractures at present. Lu et al reported a study on the curative effect of Zengye Chengqi Decoction on abdominal distension and constipation after thoracolumbar compression fracture, taking Zengye Chengqi Decoction can improve flatus, stool improved thoracolumbar compression fractures patients with symptoms of gastrointestinal dysfunction and its clinical efficacy is better than conventional Western medicine treatment, it can be faster, better recovery of gastrointestinal function. $^{24}$ But this study was not a study on prophylactic use of drugs. Zhang treated 60 cases of constipation after acute thoracolumbar compressibility fracture by orally adding liquid Chengqi Decoction combined with its preparation ion transduction, and achieved good curative effect. ${ }^{25}$ This is the first study using Zengye Chengqi Decoction as a preventive factor for constipation after thoracolumbar fractures, which is different from other studies.

There are some limitations in this study. First, other factors that might affect constipation, such as meals at the hospital, ambulation situation, constipation history, and daily activities, were not analyzed in this study as this information was not available in the medical records of all patients. Second, all patients included in this study were tested for neurological deficits and the results showed that their neurological function was normal. Further investigations are needed to explore whether neurological deficits would be a risk factor for TVCF patients. Third, the sample size of this study was relatively small. Multi-center studies with a large sample size are needed to validate the results of this study. Fourth, there is no analysis on the degree of fracture affected the constipation which need a further investigation.

\section{Conclusion}

In this study, we retrospectively reviewed the constipation status of elderly patients after TVCFs. Our results showed that patients with vertebral fractures featuring a higher degree of compression may have a higher risk of constipation. The preintervention of Zengyechengqi decoction reduced the incidence of constipation after TVCFs, suggesting that Zengyechengqi decoction might be used to alleviate constipation in elderly patients after TVCFs.

\section{Data Sharing Statement}

The authors intend to share individual deidentified participant data. Data obtained in the results section: Demographic and clinical characteristic of patients with constipation after TVCF and Multivariate binary logistic regression analysis of constipation after TVCF can be shared. Emails could be sent to the address to obtain the shared data: suqiuju_336@163.com. We may balance the potential benefits and risks for each request and then provide the data that could be shared. Data will be available for two years after publication.

\section{Funding}

This work was supported by the National Natural Science Foundation (81973878, 81873320), Jiangsu Natural Science Foundation (BK20180167), Wuxi Municipal Health Planning Commission's Science and Education Project (QNRC042).

\section{Disclosure}

The authors declare that they have no competing interests.

\section{References}

1. Jin $\mathrm{C}, \mathrm{Xu} \mathrm{G}$, Weng $\mathrm{D}$, et al. Impact of magnetic resonance imaging on treatment-related decision making for osteoporotic vertebral compression fracture: a prospective randomized trial. Med Sci Monitor. 2018;24:50-57.

2. Martikos K, Greggi T, Faldini C, Vommaro F, Scarale A. Osteoporotic thoracolumbar compression fractures: long-term retrospective comparison between vertebroplasty and conservative treatment. Eur Spine J. 2018;27(Suppl 2):244-247. doi:10.1007/s00586-018-5605-1

3. Cha KH, Cho TG, Kim CH, et al. Comparative study on the period of absolute bed rest of vertebral compression fracture. Korean $J$ Spine. 2013;10(3):144. doi:10.14245/kjs.2013.10.3.144

4. Rui C. 86 Cases Study: treatment of Stroke Constipation with ZengYeChengQi Decoction (ZYCQD) and BuYangHuan Wu Decoction (BYHWD. Int J Trad Chinese Med. 2009;31(2):139-149.

5. Liang X, Zhou M, Ge XY, et al. Efficacy of traditional Chinese medicine on sepsis: a systematic review and meta-analysis. Int $J$ Clin Exp Med. 2015;8(11):20024-20034.

6. Li D. The Clinical Observation of Modiifed ZengYeChengQi Decoction Combined with Deanxit in Treating Qi Stagnation and Intestinal Dryness Syndrome of Constipation-Predominant Irritable Bowel Syndrome.

7. Mauch JT, Carr CM, Cloft H. Review of the Imaging features of benign osteoporotic and malignant vertebral compression fractures. Am J Neuroradiol. 2018;39:9. doi:10.3174/ajnr.A5528

8. Sobrado CW, José Felippe I Neto C, et al. Diagnosis and treatment of constipation: a clinical update based on the Rome IV criteria. J Coloproctol. 2018;38:2. 
9. State Administration of Traditional Chinese Medicine. Diagnostic andTherapeutic Criteria for TCM Diseases. Nanjing: Nanjing University Press; 2012:14.

10. Lv Z, Liu JX, Zhang P, et al. Clinical effect of extra early operation combined with zengye chengqi decoction in treatment of patients with hypertensive cerebral hemorrhage. Med Pharm J Chin Peoples Liberation Army. 2015. Available from: https://d.wanfangdata.com. cn/periodical/hbgfyy201506021. Accessed July 27, 2021.

11. Yanfen Y, Jinhua Z. Research Progress of Clinical Application of Zengye Chengqi Decoction. Chinese Medicine Modern Distance Education of China; 2014

12. Fu GL, Liao MF, Yu GZ, et al. Clinical effect ofheat sensitive moxibustion treating abdominal distention and constipation in patients with lumbar vertebral compression fracture. China Modern Med. 2015. Available from: http://en.cnki.com.cn/Article_en/ CJFDTOTAL-ZGUD201515032.htm. Accessed July 27, 2021.

13. Wang Q-X. Therapeutic observation of needle embedding for constipation after thoracolumbar vertebral fracture. J Acupuncture Tuina Sci. 2016;14(3):207-210. doi:10.1007/s11726-016-0926-2

14. Jing D, Jia L. Assessment of patients' psychological state and selfefficacy associated with postoperative constipation after thoracolumbar fracture surgery. J Int Med Res. 2019;47(9):4215-4224. doi: $10.1177 / 0300060519859732$

15. Alexandru D, So W. Evaluation and management of vertebral compression fractures. Perm J. 2012;16(4):46-51. doi:10.7812/ TPP/12-037

16. Donnally J III, DiPompeo CM, Varacallo M. Vertebral Compression Fractures Chester; 2019.

17. Lee HJ, Seo JC, Park SH, et al. Acupuncture in patients with a vertebral compression fracture: a protocol for a randomized, controlled, pilot clinical trial. J Pharmacopuncture. 2015;18(1):79-85. doi:10.3831/KPI.2015.18.009
18. Wang Z, Wang XQ, Zhang Z, et al. Effects of zengyi chengqi decoction on intestinal tissue structure, serum il-6 and TNF - levels in rats with hot intestinal syndrome. J Animal Sci Veterinary Med. 2016;47(4):828-835.

19. Peng Y, Wang P, Cheng J, Zhang C, Tian D. Effect of Zengye Chengqi Decoction on oxidative stress in elderly model of constipation. New Trad Chinese Med. 2013;45(03):164-167.

20. Jiang H, Deng S. Effect of Zengye Chengqi decoction on the regulation of neuropeptide secretion in rats with intestinal microflora disorder. Sichuan Tradi Chinese Med. 2018;36(10):46-48.

21. Xin H, Zhang J. Clinical observation on the therapeutic effect of zengyi chengqi decoction on senile functional constipation. Sichuan Trad Chinese Med. 2008;03:58-59.

22. Zhang C-J. 75 cases of constipation after hip fracture treated by jiawei-zeng liquid cheng-qi decoction. Chinese Sci Technol Trad Chinese Med. 2004;2:76.

23. Chen T. Clinical observation on the treatment of opioid-induced constipation with zengyi chengqi decoction. J Pract Trad Chinese Med. 2016;32(11):1061.

24. Lu Y, Zhou L, Xie L, Tao Y. Study on the therapeutic effect of Zengye Chengqi Decoction on abdominal distension and constipation after thoracolumbar compression fracture. J Liaoning Univ Trad Chinese Med. 2017;19(04):66-68.

25. Zhang. A summary of 60 cases of constipation after acute thoracolumbar compression fracture treated with Zengye Chengqi Decoction. Hunan J Trad Chinese Med. 2017;33(08):89-90.
International Journal of General Medicine

\section{Publish your work in this journal}

The International Journal of General Medicine is an international, peer-reviewed open-access journal that focuses on general and internal medicine, pathogenesis, epidemiology, diagnosis, monitoring and treatment protocols. The journal is characterized by the rapid reporting of reviews, original research and clinical studies across all disease areas. The manuscript management system is completely online and includes a very quick and fair peer-review system, which is all easy to use. Visit http://www.dovepress.com/ testimonials.php to read real quotes from published authors. 